\title{
A simulation study of the effect of drift electric fields on the response of radiation detectors using the PENELOPE code
}

\author{
L.M.N. Távora ${ }^{a, b, *}$, T.H.V.T. Dias ${ }^{\mathrm{b}}$, C.A.N. Conde ${ }^{\mathrm{b}}$ \\ ${ }^{a}$ E.S.T.G., Instituto Politécnico de Leiria, Morro do Lena-Alto do Vieiro, 2411-901 Leiria, Portugal \\ ${ }^{\mathrm{b}}$ Centro de Instrumentação, Dep. Física da Universidade de Coimbra, Coimbra, Portugal
}

Received 2 November 2004; received in revised form 5 February 2006; accepted 20 February 2006

Available online 29 March 2006

\begin{abstract}
The effect of the presence of a drift electric field on the response of gaseous and semiconductor radiation detectors to energetic X-rays (energies $\mathscr{E}_{\text {ph }}$ from 20 to $200 \mathrm{keV}$ ) is investigated using the PENELOPE code to simulate the photo-absorption and the slow-down of the electrons produced in $\mathrm{Si}, \mathrm{Ge}$, and Xe gas at $1 \mathrm{~atm}$. For typical drift fields, the energy $\mathscr{E}_{\mathrm{d}}$ deposited in the detection media is calculated taking into account the energy exchanged by the electrons with the field. The analysis of the calculated $\mathscr{E}$ d distributions shows that the effect of the field on the distributions is negligible in $\mathrm{Si}$ and $\mathrm{Ge}$ semiconductor detectors, but not in Xe gas detectors, where for $E / p=0.8 \mathrm{~V} \mathrm{~cm}^{-1} \mathrm{Torr}^{-1}$ the fluctuations introduced by the field for $\mathscr{E} \mathrm{ph} \approx 180 \mathrm{keV}$ approach the intrinsic values for Xe, and the intrinsic discontinuity in linearity when $\mathscr{E}_{\mathrm{ph}}$ crosses the Xe $K$-edge $(34.56 \mathrm{keV})$ is further reduced by $\approx 4 \%$. The simulation data also suggest that this field effect may cause some deviations to the expected Gaussian response of Xe detectors to the absorption of monoenergetic photons.
\end{abstract}

(C) 2006 Elsevier B.V. All rights reserved.

PACS: 29.40.-n; 29.40.Cs; 29.40.Wk; 29.30.Kv

Keywords: Gas detectors; Semiconductor detectors; X-ray and gamma-ray spectra distortion; Electric-field effects; Monte Carlo simulation

\section{Introduction}

Most detection devices for X-rays are based on the fact that X-rays interact with matter producing electrons which dissipate their energy along numerous inelastic and elastic collisions, until all reach sub-ionization energies [1]. The number $n$ of charge carriers produced depends on the photon and electron interactions in the detector absorbing medium, so the mean energy $w$ to produce a charge carrier $\left(w=\mathscr{E}_{\mathrm{ph}} / n\right.$, where $\mathscr{E}_{\mathrm{ph}}$ is the X-ray energy) is characteristic of that medium, and may show some dependence on $\mathscr{E}_{\mathrm{ph}}$ [2]. The mean number $n$ is a good measure of $\mathscr{E}_{\mathrm{ph}}$ when the detector intrinsic response is linear, i.e., when $w$ is approximately constant and $n$ is proportional to $\mathscr{E}_{\mathrm{ph}}$. Studies of intrinsic energy linearity

\footnotetext{
${ }^{*}$ Corresponding author. E.S.T.G., Instituto Politécnico de Leiria, Morro do Lena-Alto do Vieiro, 2411-901 Leiria, Portugal.

E-mail address: lmtavora@estg.ipleiria.pt (L.M.N. Távora).
}

and energy resolution of X-ray detectors can be found in Refs. [2-5] where the dependence on $\mathscr{E}$ ph, characteristic discontinuities and asymmetry effects observed in detector response are explained in terms of the shell-structure of the atoms in the absorbing medium.

Drift fields with intensities below the threshold for inelastic scattering of the electrons in the detection medium are often applied in a detector to guide the cloud of subionization electrons towards a collecting electrode or an amplification stage. When travelling along the detector's drift region under such a field, electrons will not reach the ionization threshold and will not multiply, because energy gains from the field are not high enough to compensate the losses in elastic collisions.

However, the absorption region and the drift region of a detector are often one and the same volume, and it is commonly assumed that the drift-field will not influence the energy-deposition process. This assumption, though, overlooks the fact that during slow-down some charge 
carriers go through a wide range of high energies, well above the ionization threshold, so that the energy these fast electrons exchange with the field in their long ranges will result in extra ionization events. This changes the fluctuations and the number of charge carriers produced per photon in the medium, and may visibly alter the response of the detector compared to the case when no field is present during slow-down.

We first noticed this subtle field effect in a detailed Monte Carlo study of the $w$-value, Fano factor $F$, and intrinsic energy resolution in $\mathrm{Xe}$ for the absorption of electron beams with energies $\mathscr{E}_{\mathrm{e}}$ up to $200 \mathrm{keV}$ [6], where we encountered an unexpected dependence of $F$ and $w$ on the applied drift field. For the absorption in Xe of X-rays with energies $\mathscr{E}_{\mathrm{ph}}$ in the range $60-200 \mathrm{keV}$, we later analysed in Ref. [7] the effect of the drift field using the PENELOPE code [8] to simulate the photo-absorption and the electron trajectories during slow-down, bringing to evidence a fieldrelated broadening, shift, and distortion of the calculated energy peaks at increasing $\mathscr{E}_{\mathrm{ph}}$ and applied drift field $E$.

In the present work, we use PENELOPE to extend this study to Ge and Si semiconductor detectors, and to further examine the effect of the drift field in Xe gas. Apart from radiation detectors, we note that Refs. $[9,10]$ report the influence of a drift field on the response of ionization chambers. In the meantime, techniques to overcome this field effect have been explored in Ref. [11].

\section{Simulation method}

The PENELOPE software package $[8,12]$ is a versatile and powerful code which can simulate the transport of electrons/positrons and photons with energies $\mathscr{E}$ from $1 \mathrm{GeV}$ to a few hundred $\mathrm{eV}$ for user-defined absorbing media and detector geometries, keeping track of the energy $\mathscr{E}_{\mathrm{d}}$ deposited in the detector during the absorption and energy degradation process. For charged particles, PENELOPE uses the condensed history technique and a class II algorithm [13], where the simulation of events involving energy transfers above given thresholds is combined with the continuous slowing down approximation (CSDA). We note that, in the absence of an electric field, PENELOPE gives $\mathscr{E}_{\mathrm{d}}=\mathscr{E}$ when no loss of charge carriers or radiation occurs, and distributions of $\mathscr{E}_{\mathrm{d}}$ will be $\delta$-functions in these circumstances.

In the present study, X-ray photons with energies $\mathscr{E}_{\mathrm{ph}}$ in the range $20-200 \mathrm{keV}$ are absorbed in a detection thickness of $L=5 \mathrm{~mm}$ in Ge and Si and $L=5 \mathrm{~cm}$ in Xe gas at $1 \mathrm{~atm}$, and the photo-electron and secondary electrons are followed down to kinetic energies of $150 \mathrm{eV}$ or until they cross the boundary at $L$. The chosen $L$ lengths are large enough to reduce radiation and electron losses to a negligible level, and the cutoff at $150 \mathrm{eV}$ is low enough to guarantee that exchanges with the field beyond this point are also negligible. The drift fields used in the calculations are $E=600 \mathrm{~V} \mathrm{~cm}^{-1}$ in the $\mathrm{Si}$ and $\mathrm{Ge}$ semiconductor media and $E / p$ in the range $0.1-0.8 \mathrm{~V} \mathrm{~cm}^{-1} \mathrm{Torr}^{-1}$ in $\mathrm{Xe}$ gas
$\left(E=600 \mathrm{~V} \mathrm{~cm}^{-1}\right.$ in the semiconductors and $E / p=$ $0.8 \mathrm{~V} \mathrm{~cm}^{-1} \mathrm{Torr}^{-1}$ in Xe are higher field limits just below the thresholds for inelastic scattering [14,15]).

\section{Results and discussion}

To examine the effect of the drift field, PENELOPE calculates distributions of the deposited energy $\mathscr{E}_{\mathrm{d}}$ which includes the energy the electrons exchange with the field during slow-down. We note that the profile and behaviour of the distributions will reflect the effect of the drift field alone, since the distributions will be $\delta$-functions $\left(\mathscr{E}_{\mathrm{d}}=\mathscr{E}_{\mathrm{ph}}\right)$ at zero field.

In Fig. 1(a)-(c), the continuous curves are the $\mathscr{E}_{\mathrm{d}}$ distributions obtained for $100 \mathrm{keV}$ X-rays in Xe gas, Ge and $\mathrm{Si}$, for the drift fields indicated. The field effect is clear, and the widths of the peaks measure the fluctuations in $\mathscr{E}_{\mathrm{d}}$ introduced by the field. The effect is far more relevant in Xe $($ spread $\approx 400 \mathrm{eV})$ than in $\mathrm{Si}$ or $\mathrm{Ge}($ spreads $\approx 3 \mathrm{eV})$. A similar trend was found for $\mathscr{E}_{\mathrm{ph}}$ ranging from 20 to $200 \mathrm{keV}$. This behaviour is easily understood in terms of electron mean free-paths, which are about four orders of magnitude larger in Xe at $1 \mathrm{~atm}$ than in the semiconductor media, see Fig. 2, and longer paths and ranges involve higher energy exchanges with the field. On the other hand, the peaks in Fig. 1(a)-(c) can be compared with the corresponding intrinsic peaks (dashed curves), whose widths reflect the fluctuations in the number $n$ of subionization charge carriers/electrons produced per photon of energy $\mathscr{E}_{\mathrm{ph}}$ absorbed in each medium. These are Gaussian curves with full-width at half-maximum $\Gamma_{\text {int }}=2.35\left(F w \mathscr{E}_{\mathrm{ph}}\right)^{1 / 2}$, where the Fano factor $F$ and the $w$-value for the given $\mathscr{E}_{\mathrm{ph}}$ are given in Fig. $1\left(F=\sigma^{2} / n\right.$ is the relative variance in $n$, and $w=\mathscr{E}_{\mathrm{ph}} / n$ is the mean energy required to produce a sub-ionization electron $[1,2])$. Comparing Fig. 1(a) with Figs. 1(b) and (c), we observe that while in Xe the field-related spread in $\mathscr{E}_{\mathrm{d}}$ is of the same order of the fluctuations in the intrinsic peak, in $\mathrm{Si}$ and $\mathrm{Ge}$ the field contributions are two to three orders of magnitude lower. In view of these results, we can conclude that the response of $\mathrm{Si}$ or $\mathrm{Ge}$ detectors is not expected to be sensitive to the effect of the applied drift field.

For Xe gas detectors the effect is more important, and in addition to the results presented in Ref. [7] we now examine further aspects of the drift field effect on the energy response in $\mathrm{Xe}$ and present a systematic discussion, analysing the width, position, and asymmetry of the $\mathscr{E}_{\mathrm{d}}$ distributions in Xe gas as a function of $\mathscr{E}_{\mathrm{ph}}$.

In Fig. 3(a), the full-width at half-maximum $\Gamma$ of Gaussian fits to the calculated $\mathscr{E}_{\mathrm{d}}$ distributions in Xe are shown for three reduced drift fields $E / p$, together with the curves $\Gamma_{\text {int }}$ for the intrinsic distributions. For the three $E / p$ values, the spread $\Gamma$ of the $\mathscr{E}_{\mathrm{d}}$-peaks is seen to increase in general with $\mathscr{E}_{\text {ph }}$, because faster electrons have longer paths in the gas (see Fig. 2), and the energy exchanges with the field will accordingly increase. The effect becomes naturally more important at higher applied electric fields, and we 

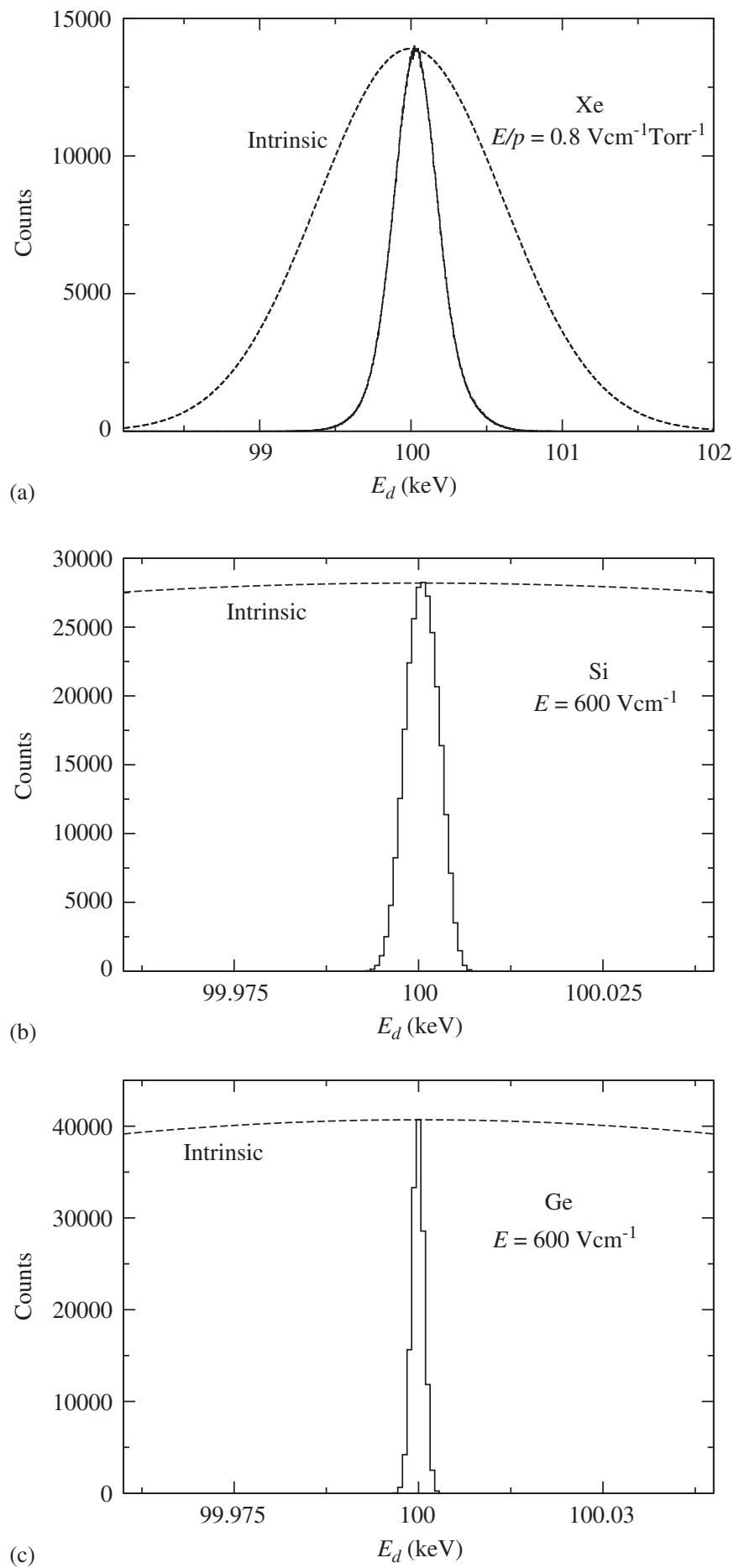

Fig. 1. Calculated distributions of the energy $\mathscr{E}_{\mathrm{d}}$ deposited (a) in Xe gas at $1 \mathrm{~atm}$; (b) in Si and (c) in Ge, for $\mathscr{E}_{\mathrm{ph}}=100 \mathrm{keV}$ X-rays and for the drift fields $E$ indicated. The dashed curves are the intrinsic distributions, which are Gaussians with full-widths at half-maximum $\Gamma_{\text {int }}=2.35 \sqrt{F w \mathscr{E} \text { ph. }}$. To obtain $\Gamma_{\text {int }}$, we used the values $F=0.080, w=2.96 \mathrm{eV}$ in $\mathrm{Ge}[1]$ and $F=0.084, w=3.76 \mathrm{eV}$ in $\mathrm{Si}[1]$. In Xe, the value for $\Gamma_{\text {int }}$ was taken from Fig. $3\left(\Gamma_{\text {int }}=1.5 \mathrm{keV}\right.$ at $\left.100 \mathrm{keV}\right)$.

observe in Fig. 3(a) that for $E / p=0.8 \mathrm{~V} \mathrm{~cm}^{-1} \mathrm{Torr}^{-1}$ and photon energies above $\mathscr{E}_{\mathrm{ph}} \approx 180 \mathrm{keV}$ the fluctuations $\Gamma$ in $\mathscr{E}_{\mathrm{d}}$ even exceed the intrinsic fluctuations $\Gamma_{\text {int }}$. We note that, although the effect is small for the lower $\mathscr{E}_{\mathrm{ph}}$,

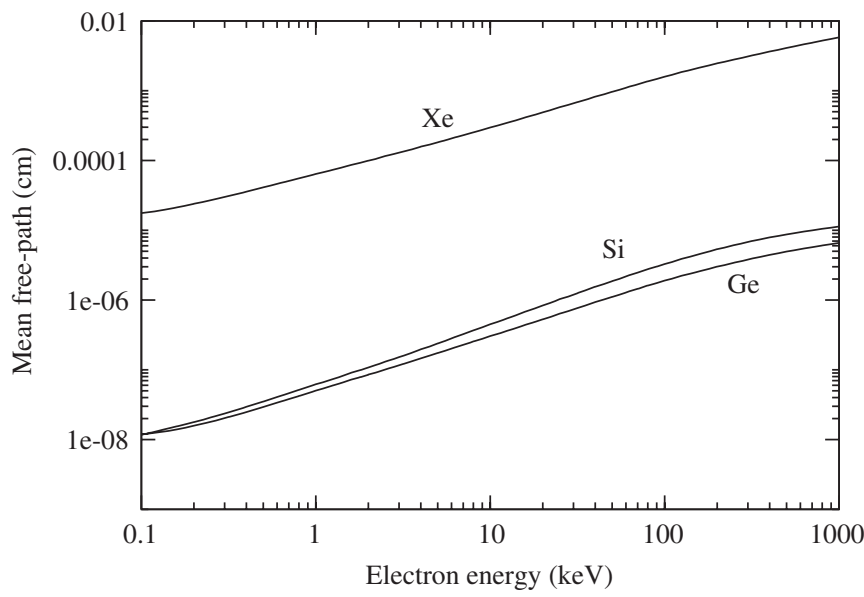

Fig. 2. Electron mean free-paths in $\mathrm{Ge}$ and $\mathrm{Si}$, and in $\mathrm{Xe}$ gas at $p=1 \mathrm{~atm}$, obtained using the electron scattering cross-sections from the EEDL database [16].
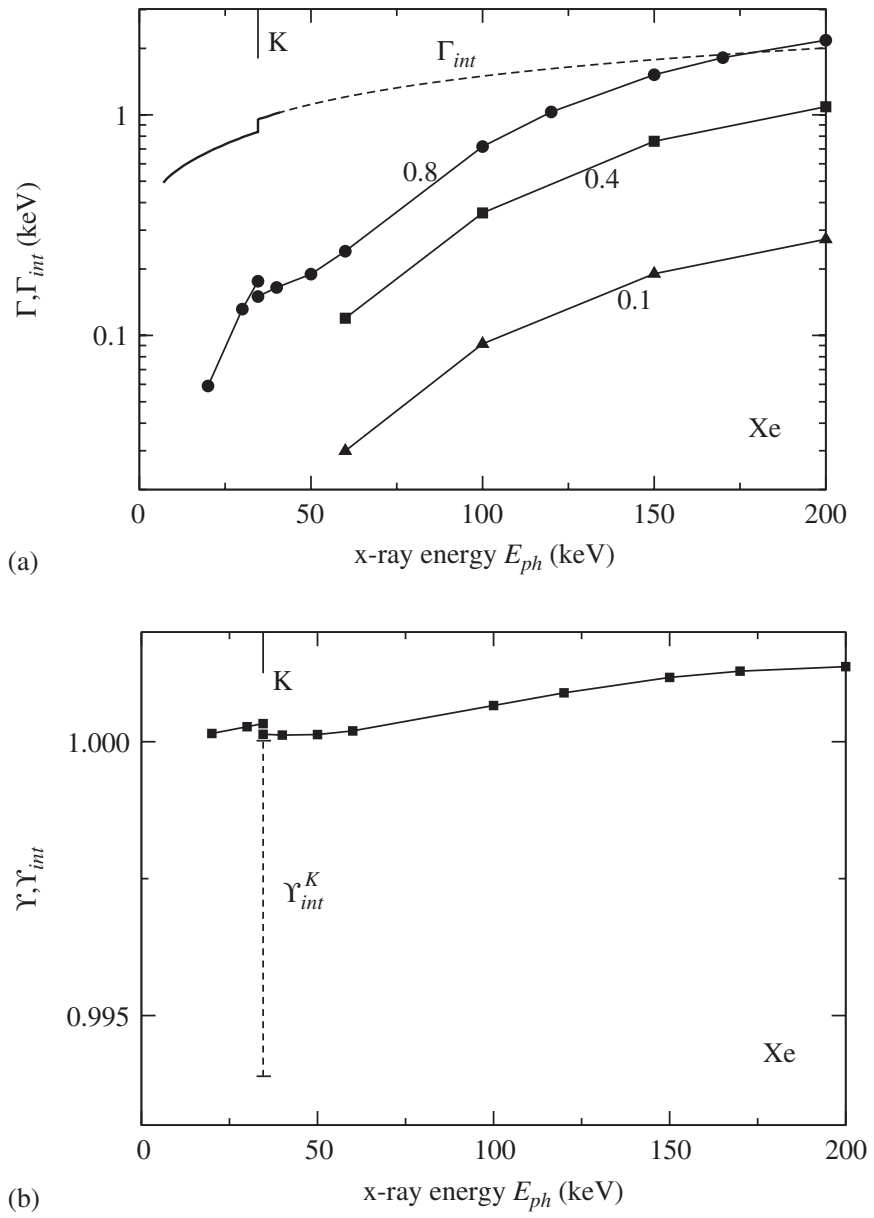

Fig. 3. (a) Full-width at half-maximum $(\Gamma)$ of the Gaussian fits to the calculated distributions of the energy $\mathscr{E}_{\mathrm{d}}$ deposited in Xe gas as a function of X-ray energy $\mathscr{E}_{\mathrm{ph}}$ for $E / p=0.1,0.4$ and $0.8 \mathrm{~V} \mathrm{~cm}^{-1} \mathrm{Torr}^{-1}$, together with the curve for the intrinsic values $\Gamma_{\text {int }}=2.35\left(F w \mathscr{E}_{\mathrm{ph}}\right)^{1 / 2}$, (for $\mathscr{E}_{\mathrm{ph}}<41 \mathrm{keV}$, the $\mathscr{E}_{\mathrm{ph}}$-dependent $F$ - and $w$-values from Ref. [2] were used; for higher $\mathscr{E}_{\mathrm{ph}}$ (dashed region), the curve $\Gamma_{\text {int }}$ was extrapolated assuming at $200 \mathrm{keV}$ the values $F=0.17$ and $w=21.5 \mathrm{eV}$ from Ref. [1]). (b) Ratio $\Upsilon=\mathscr{E}_{\mathrm{dm}} / \mathscr{E}_{\mathrm{ph}}$ for $E / p=0.8 \mathrm{~V} \mathrm{~cm}^{-1} \mathrm{Torr}^{-1}$, where $\mathscr{E}_{\mathrm{dm}}$ is the mean value of $\mathscr{E}_{\mathrm{d}}$. The dashed vertical bar $Y_{\text {int }}^{K}$ shows the intrinsic discontinuity in linearity [2]. The Xe $K$-edge ( $\left.\mathscr{E}_{K}=34.56 \mathrm{keV}\right)$ is indicated at the top in (a) and (b). 
a discontinuity in $\Gamma$ is visible when $\mathscr{E}_{\text {ph }}$ crosses the Xe $K$-edge, which is about $\approx 4 \%$ of the intrinsic discontinuity in $\Gamma_{\text {int }}$ observed in the same region. This discontinuous behaviour has been explained previously in terms of atomic-shell effects in the production of electrons whenever a new (inner) shell becomes energetically available to photo-ionization [1-5].

Together with the increase in peak-width at increasing $\mathscr{E}_{\mathrm{ph}}$, the results show that the mean value $\mathscr{E}_{\mathrm{dm}}$ of the $\mathscr{E}_{\mathrm{d}}$ distributions in $\mathrm{Xe}$ is always higher than $\mathscr{E}_{\mathrm{ph}}$ and shifts gradually towards higher values, as $\mathscr{E} \mathrm{ph}$ increases. For the higher field used in the calculations $\left(E / p=0.8 \mathrm{~V} \mathrm{~cm}^{-1} \mathrm{Torr}^{-1}\right)$, this shift is shown in Fig. 3(b) by the curve representing the ratio $\Upsilon=\mathscr{E}_{\mathrm{dm}} / \mathscr{E}_{\mathrm{ph}}$ as a function of $\mathscr{E}_{\mathrm{ph}}$. A discontinuity in $\Upsilon$ is also present, although smaller than the intrinsic discontinuity $Y_{\mathrm{int}}^{K}$, represented in Fig. 3(b).

The simulation data also show that the $\mathscr{E}_{\mathrm{d}}$ distributions slightly deviate from Gaussian shapes. This is shown in Fig. 4(a) for $60 \mathrm{keV}$ photons absorbed in Xe under a drift

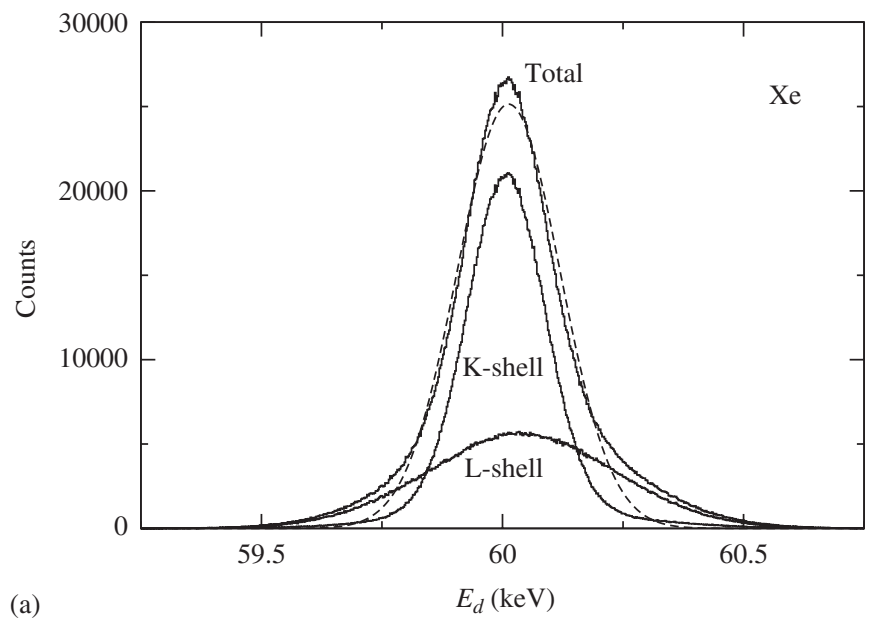

(a)

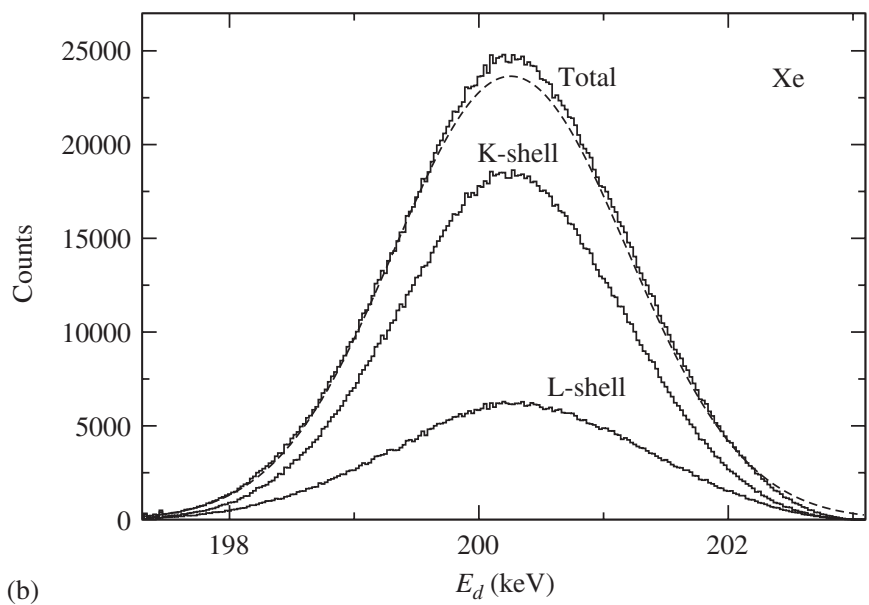

Fig. 4. Distributions of the energy $\mathscr{E}_{\mathrm{d}}$ deposited in Xe gas under a drift field $E / p=0.8 \mathrm{~V} \mathrm{~cm}^{-1} \mathrm{Torr}^{-1}$, calculated for X-ray photons with energies (a) $\mathscr{E}_{\mathrm{ph}}=60 \mathrm{keV}$ and (b) $\mathscr{E}_{\mathrm{ph}}=200 \mathrm{keV}$ showing the partial contributions from $K$ - and $L$-photo-ionization events to the total peak. The dotted curves are Gaussian fits to the total peaks. field of $E / p=0.8 \mathrm{~V} \mathrm{~cm}^{-1} \mathrm{Torr}^{-1}$, where the $\mathscr{E}_{\mathrm{d}}$ distribution is asymmetric and fails to be properly represented by a Gaussian curve. These effects are associated with the fact that photo-ionization events occur from different $\mathrm{Xe}$ atomic shells, mostly $K$ and $L$, yielding electrons with different initial energies which thus experience different field effects. This is the case for $\mathscr{E}_{\mathrm{ph}}=60 \mathrm{keV}$ in Fig. 4(a), where the (approximately Gaussian) $K$ - and $L$-contributions to the total peak have different widths as well as distinct positions (distinct shifts above $\mathscr{E}_{\mathrm{d}}=\mathscr{E}_{\mathrm{ph}}$ ), and this causes the asymmetry in the total peak. However, for $\mathscr{E}_{\mathrm{ph}} \gg \mathscr{E}_{K}$, e.g., for $\mathscr{E}_{\mathrm{ph}}=200 \mathrm{keV}$ in Fig. 4(b), the energies of the electrons and the positions of the partial peaks belonging to $K$ - and $L$-events get closer, and the asymmetry is reduced. We note that the field-related distortion will add to the intrinsic distortion discussed in Ref. [2], which is also caused by atomic-shell effects.

\section{Acknowledgements}

This work was carried out at Escola Superior de Tecnologia e Gestão (IPLeiria) and in the Departamento de Física da Universidade de Coimbra (Research Unit No. 217/94). The authors acknowledge financial support from FEDER, Fundação para a Ciência e Tecnologia (through Project POCTI/FP/FNU/50222/2003).

\section{References}

[1] G.F. Knoll, Radiation Detection and Measurement, third ed., Wiley, New York, 2000

[2] T.H.V.T. Dias, J.M.F. dos Santos, P.J.B.M. Rachinhas, F.P. Santos, C.A.N. Conde, A.D. Stauffer, J. Appl. Phys. 82 (1997) 2742.

[3] T.H.V.T. Dias, F.P. Santos, J.M.F. dos Santos, P.J.B.M. Rachinhas, C.A.N. Conde, A.D. Stauffer, IEEE Trans. Nucl. Sci. NS-43 (1996) 1432.

[4] C.A.N. Conde, Gas scintillation proportional counters for X-ray spectrometry, in: K. Tsuji, J. Injuk, R. Van Grieken (Eds.), X-Ray Spectrometry: Recent Technological Advances, Wiley, New York, 2004.

[5] G.W. Fraser, A.F. Abbey, A. Holland, K. McCarthy, A. Owens, A. Wells, Nucl. Instr. and Meth. A 350 (1994) 368;

A. Owens, G.W. Fraser, A.F. Abbey, A. Holland, K. McCarthy, A. Keay, A. Wells, Nucl. Instr. and Meth. A 382 (1996) 503.

[6] P.J.B.M. Rachinhas, T.H.V.T. Dias, F.P. Santos, C.A.N. Conde, A.D. Stauffer, IEEE Trans. Nucl. Sci. NS-46 (1999) 1898.

[7] L.M.N. Távora, C.A.N. Conde, F.P. Santos, T.H.V.T. Dias, P.J.B.M. Rachinhas, Rad. Phys. Chem. 7 (2004) 723.

[8] F. Salvat, J.M. Fernández-Varea, J. Sempau, E. Acosta, PENELOPE, a code system for Monte Carlo simulation of electron and photon transport, OECD/NEA 5-7 November 2001, NEA/NSC/ DOC, 2001.

[9] N. Takata, T. Matsumoto, Nucl. Instr. and Meth. A 302 (1991) 327.

[10] N. Takata, T. Sugita, in: J.A. Seibert (Ed.), Conference Record of the 1999 Nuclear Science Symposium, 24-30 October 1999, Seattle, USA, vol. 2, 1999, p. 638.

[11] P.N.B. Neves, L.M.N. Távora, C.A.N. Conde, in: J.A. Seibert (Ed.), Conference Record of the 2004 Nuclear Science Symposium, 16-22 October 2004, Rome, Italy, vol. 1, 2004, p. 543

[12] J. Sempau, J.M. Fernández-Varea, E. Acosta, F. Salvat, Nucl. Instr. and Meth. B 207 (2003) 107. 
[13] M.J. Berger, Meth. Comput. Phys. 1 (1963) 135.

[14] Modular Pulse-Processing Electronic and Semiconductor Radiation Detectors, EG\&G Ortec. Available from 〈http://www.ortec-online.com/〉.

[15] F.P. Santos, T.H.V.T. Dias, A.D. Stauffer, C.A.N. Conde, J. Phys. D: Appl. Phys. 27 (1994) 42.
[16] S.T. Perkins, D.E. Cullen, S.M. Seltzer, Tables and graphs of electron-interaction cross sections from $10 \mathrm{eV}$ to $100 \mathrm{GeV}$ derived from the LLNL Evaluated Electron Data Library (EEDL), $\mathrm{Z}=1-100$, Lawrence Livermore National Laboratory Report UCRL-50400, vol. 31, 1991. 